\title{
Hash Table Design and Optimization for Software Virtual Switches
}

\author{
Yipeng Wang, Sameh Gobriel, Ren Wang, \\ Tsung-Yuan Charlie Tai \\ Intel Labs, Intel Corporation \\ [yipeng1.wang,sameh.gobriel,ren.wang,charlie.tai] \\ @intel.com
}

\begin{abstract}
Flow classification is a common first step in various virtual network functions (VNFs), Software Defined Networking (SDN) applications, as well as network infrastructure components including virtual switches and routers. Software flow classification often employs hash table based lookup mechanisms, where a key constructed from an input packet is looked up across the different rules stored in the table and the corresponding action (e.g., forward, encapsulate, etc.) is retrieved. In this paper we analyze, in depth, various hash table design options and optimizations used in the state-of-the-art virtual switches, and how hardware resources impact the performance. Based on the understanding, we summarize the pros and cons of different designs, and provide insights toward further optimizations The understanding gained through our analysis also sheds lights on how to design optimal hash tables for flow classification for various use cases.
\end{abstract}

\section{CCS CONCEPTS}

- Networks $\rightarrow$ Routers; Bridges and switches; Network servers; Data path algorithms; Packet classification; Network performance analysis; $\bullet$ Theory of computation $\rightarrow$ Bloom filters and hashing;

\section{ACM Reference Format:}

Yipeng Wang, Sameh Gobriel, Ren Wang, Tsung-Yuan Charlie Tai and Cristian Dumitrescu. 2018. Hash Table Design and Optimization for Software Virtual Switches. In KBNets'18: ACM SIGCOMM 2018 Afternoon Workshop on Kernel Bypassing Networks , August 20, 2018, Budapest, Hungary. ACM, New York, NY, USA, 7 pages. https://doi.org/10.1145/3229538.3229542

\section{INTRODUCTION}

Software Defined Networking (SDN) paradigm is a fundamental shift in the way network has been designed, configured, and deployed. The SDN framework tends to make the data plane completely programmable and separated from the control logic. SDN introduces a centralized control structure which dynamically configures and governs all underlying hardware based on end user application requirements. Network Function Virtualization (NFV), on

Permission to make digital or hard copies of all or part of this work for personal or classroom use is granted without fee provided that copies are not made or distributed for profit or commercial advantage and that copies bear this notice and the full citation on the first page. Copyrights for components of this work owned by others than ACM must be honored. Abstracting with credit is permitted. To copy otherwise, or republish, to post on servers or to redistribute to lists, requires prior specific permission and/or a fee. Request permissions from permissions@acm.org.

KBNets'18, August 20, 2018, Budapest, Hungary

(c) 2018 Association for Computing Machinery.

ACM ISBN 978-1-4503-5909-2/18/08 . \$ \$15.00

https://doi.org/10.1145/3229538.3229542

\author{
Cristian Dumitrescu \\ Network Platforms Group, Intel Corporation \\ cristian.dumitrescu@intel.com
}

the other hand, allows network functions (e.g., packet forwarding and dropping) to be performed in virtual machines (VMs) running on general purpose computing hardware in a cloud infrastructure rather than on dedicated devices. NFV entails implementing network functions -currently available on proprietary middle boxes and network equipment hardware- in software. Such Virtual Network Functions (VNFs) are deployed on commodity servers allowing service providers to leverage various virtualization and cloud technologies.

Virtual switches and routers ${ }^{1}$ are widely deployed in SDN networks to forward packets between different VNFs running inside VMs on the same platform or across the network on different platforms. Basically they are software applications with similar functionalities to traditional hardware switches and routers. When a packet is received on one of interface ports (either received from a VM or from the physical network), the main functionality is to forward this packet to the correct output port given the packet header information. Comparing to hardware switches, software virtual switches are more flexible while performance has usually been a concern. However, with the recent development of kernelbypass technologies the performance gap is narrowing and software switches have become the defacto standard in all SDN deployments.

While kernel-bypass alleviates the I/O cost significantly, packet processing itself becomes one of the major bottlenecks in virtual switches. In software packet processing, hash table plays a very important role. They are used for various purposes: ACL tables, tuple-based flow classification, connection tracking, flow caches, etc. Modern virtual switches with kernel-bypass spend significant time in hash table lookup and updates. In this paper, we focus on analyzing various hash table designs and generalize design guidelines to benefit network architects and virtual switch designers.

A hash table as a concept is not new, it is rather a simple and straightforward data structure that has been classically researched and studied. Various research work (e.g. [5, 7, 10, 14, 15]) has proposed different designs and optimizations of the hash table algorithms along the years. Some works $[2,12,21]$ particularly focus on improving hash table algorithms for networking usages. However, to the best of our knowledge, not much work has systematically compared among various hash table designs and their use cases in real life virtual switch applications.

We believe that hash table components have a huge impact on networking applications and VNFs' performance, especially virtual switches with kernel-bypass, and we argue that it should be carefully designed with the underlying hardware resources in mind

\footnotetext{
${ }^{1}$ We use "virtual switch" and "virtual router" interchangeably in this paper
} 
so the optimum performance of the various network functions can be achieved.

\section{HASH TABLE IMPLEMENTATIONS}

As previously mentioned, hash tables provide a useful data structure for many network applications. When designing a hash table, three performance metrics are key factors for design evaluation to determine if it is optimal for a certain application. The metrics are:

Lookup Throughput. This is usually the most important metric as lookup is on the critical data path of packet processing. This metric reflects how fast a lookup can be performed on the hash table, that is, given an input key, how fast a matching entry together with the corresponding data are retrieved, or a no match is returned.

Update Rate. This metric is critical for the control plane throughput, it reflects how fast a hash table can be constructed (i.e. new table) or updated (i.e. existing table), that is, given a key with the corresponding data, how fast this information can be inserted in the table as new or updated if a key match is already present.

Table Utilization. This metric is an important factor to measure hash table scalability. It reflects how effectively occupied a hash table can be (i.e. load factor, which is the total number of keys successfully inserted divided by the maximum number of supported keys) before the key insertion fails (due to too many collisions, as discussed later) and/or a complete table rehashing or table resizing is necessary.

In the next sub-section we summarize how various design options can affect these performance metrics.

\subsection{Software Hash Table Designs}

The very basic hash table data structure is composed of a big array. When insert or lookup a key, the array element index is determined by hashing the key. During insertion, a small signature of the key is usually calculated and stored in the table together with the key. The key is only compared when the signature matches during lookup. This basic scheme typically has a relatively low table utilization because of collisions among the keys, which happens when hashing two or more keys results in the same index. Once collision happens, the table has to be resized in order to accommodate all keys. Various hash table designs differ on how to resolve these collisions, resulting in different characteristics regarding the previously mentioned design metrics.

Set-Associative Hash Table [15]. This method extends the simple hash table design by allowing each index (bucket) to hold more than one key. The maximum number of keys per bucket usually is pre-defined. Table utilization is definitely better than the simple hash table design, however lookup and insertion rates could be negatively impacted. This is because a single input key has to be compared against all the keys (signatures) in the bucket. Moreover, when the number of keys in a bucket exceeds the maximum allowed, similar to the basic hash table the keys have to be dropped and/or overwritten or the whole table needs to be resized and all the keys need to be rehashed into the new table which is a costly operation.

Dynamic hash table [11]. Dynamic hash table is the set of hash tables that can grow the primary table dynamically without rehashing once collision happens. However, since networking applications with extreme speed requirement usually cannot afford frequent dynamic memory allocation, a secondary table or a pool of free buckets or entries is usually used to extend the primary hash table. These are referred as overflow tables. The overflow table is typically implemented as a linked list. If a bucket is full in the primary table, new keys will be inserted into the overflow table. Overflow table is usually designed to keep the main table small and fast while providing a guarantee of storing certain number of the keys without the need of a table resizing and rehashing. The table utilization of this design is typically good but lookup and insert rate significantly degrades in the case of high load factor because of the extra operation of accessing the overflow table (and typically walking the linked list).

Multiple Hash Functions [5]. It is another design that aims to resolve the key collisions by using multiple hash functions to map the keys into buckets. One key could be mapped to multiple buckets (primary and alternate(s)). During insertion, one key could choose the bucket with less existing keys, and during lookups all possible locations of a key are probed for a possible match. This mechanism could significantly alleviate collisions, but if not optimized carefully the lookup and insert rates can also be slowed down due to the extra hashing and probing required.

Cuckoo Hash [14]. Cuckoo hash is a hash table mechanism based on set-associative table design with two hash functions (two alternative buckets). Cuckoo hash allows a new key to evict an existing key to its alternative bucket. For example, if both bucket locations are full when key A is inserted, key B that is in one of A's buckets can be displaced to key B's alternative bucket, so that A will have an empty space to be inserted. This process is recursive, meaning key $\mathrm{B}$ could displace key $\mathrm{C}$ to its alternative bucket and so on, until all keys find their new locations. The advantage of Cuckoo hash is that the lookup cost is same as 2-hash multi-way hash table while the table utilization is improved significantly. Previous researches $[2,3,13,21]$ show that Cuckoo hash is an efficient algorithm for networking applications. The algorithms proposed in those studies are often used as reference by virtual switches to implement their own Cuckoo hash table.

Hopscotch Hash [7]. Hopscotch hash is based on similar "displacing" idea as Cuckoo hash. However, instead of displacing keys to their alternative buckets, Hopscotch hash regards one entry's neighbor entries as the alternative locations to displace keys to. For example, when key A is inserted, key B in key A's neighbor entry could be displaced to key B's neighbor entry so that key A will have an empty space in its neighborhood. The entries in a neighborhood compose of a virtual bucket. The advantage of this design is that unlike Cuckoo hash, it will not cause circular dependency. Also, there is no need to use two hash functions. The hopscotch hash in [7] has the drawback that the virtual buckets are overlapping and not always cache aligned, which could be less hardware friendly in general. Also, because of the one hash function indexing mechanism, the collision rate could be higher than the bucketized Cuckoo hash design.

Other Hash Table Designs. Other traditional hash tables include linear probing and quadratic probing. They have drawbacks such as clustering issue and low CPU cache locality. We will not go into details of these algorithms. There are more advanced implementations for some standard libraries such as c++ standard library 
and they are usually based on variants of the aforementioned implementations.

\subsection{Survey of Hash Table Designs in Virtual Switches}

As previously mentioned, hash table is one of key data structures in virtual switches. They are used for connection tracking, NAT, ACL, flow caching, GRO/GSO, etc. Implementing an effective hash table structure in virtual switch is critical. In this section, we survey some state-of-the-art virtual switches, focusing on their hash table implementations.

VPP [4]. Vector Packet Processing or VPP platform is an opensource virtual switch providing user extensibility. VPP has a modular design where the packet processing datapath is organized as graph nodes. This gives the user flexibility to implement their own nodes/plugins into VPP to extend its feature. VPP provides multiple hash table implementations. First, it has a basic hash table implementation based on a one-hash set-associative table that is dynamically resized based on utilization. Second, a slightly more advanced design which is called "mhash" that can handle arbitrary key lengths by storing keys in a separate dynamic memory location. A third implementation uses the design of an overflow table that was previously discussed to handle key collisions. Another more widely used hash table in VPP is called bi-table or bounded-index extensible hash table. This hash table is essentially a hierarchical hash table. Each entry in the first level hash table points to a second level hash table (i.e. the bucket). The second level tables can double their sizes dynamically with load factor increasing. This hash table is augmented with a small LRU cache to buffer recently accessed keys. Furthermore, VPP also recently (v.17.07) introduced another hash table type based on Cuckoo hash. Besides the basic Cuckoo algorithm, this implementation follows [13] to provide multi-writer and multi-reader capabilities.

BESS [6]. Berkeley Extensible Software Switch or BESS is a software switch developed at UC Berkeley and, similar to VPP, is modular. A flexible phython based scripting language is used to define a packet datapath connecting modules together.

In BESS, Cuckoo hash is implemented as the main hash table algorithm. By default, it is a 4-way set-associative table with two hash functions. The table size is doubled when an insertion failure occurs. The hash table only stores a signature and a pointer to the full key and data pair.

OpenContrail (now named as Tungsten Fabric) vRouter [8, 20]. The vRouter is another open source virtual switch implementation as one component of the OpenContrail (Tungsten Fabric) framework. vRouter uses hash table for multiple modules including an exact match flow-table, L2 bridge forwarding table, and MPLS tags lookup table. The main hash table design is a simple 4-way set-associative structure with an overflow table, using a linked list of overflow entries. It is worth mentioning that the full key is stored in the hash table rather than in a separate memory location. This avoids an extra cache line access during key comparison.

OVS [16]. Open vSwitch or OVS is one of the most popular virtual switches deployed in data centers. Unlike other switches, OVS is not fully modular, and all packets with various protocols will typically go through the same processing pipeline rather than different modules. Furthermore, OVS is unique as it uses a "cache-based" design. The data path consists of multiple layers of software caches from the fastest to the slowest. Caches in OVS allow for recent flows to overwrite old flows and implemented as set-associative hash tables. For non-cache hash tables such as flow tables, OVS uses 5 way set-associative Cuckoo hashing table. When the table is full (default as $85 \%$ loaded), the size is doubled, and when the table is lightly loaded (default as $20 \%$ ), the size is halved.

DPDK [1]. Data Plane Development Kit or DPDK is a widely adopted kernel-bypass library for packet processing. It provides multiple hash table libraries with different flavors to fit a range of application use cases. The main hash table library, rte_hash, is an implementation of Cuckoo hash. It uses an 8-way set-associative table structure with two hash functions. Furthermore, the implementation is optimized using specific hardware features including vector processing by Intel ${ }^{\circledR}$ AVX technology to speedup the signature comparison process in the bucket, and provides thread safety using Intel®TSX technology. Both techniques improve throughput and scalability significantly from our experience with DPDK.

Additionally two other major hash table implementations are provided by the DPDK packet framework library.

One is extendable bucket hash, which implements an overflow pool of extra buckets in addition to a 4-way set-associative table, where extra buckets are linked to a full one. The advantage of extendable bucket hash is that it provides $100 \%$ guarantee to store certain number of keys by using the linked list of buckets. In the worst case, the hash table still guarantees a capacity same as the number of entries in the overflow buckets.

Another is LRU hash table which is a simple set-associative hash with LRU replacement policy, where the entries in a bucket are ordered based on their use recency thus new key can overwrite the least recently used key. Recently (in DPDK v17.11) a new library called "membership library" is introduced. The library's objective is to provide a "set-summary" to test whether a given key belongs to a specific set or not (with a small false positive probability). One mode of this library is a hash table based set-summary. Comparing to traditional hash tables, only signatures of flows are stored. This data structure can be used to cache flows in virtual switches as described in [18] to speed up lookups for recent flows.

Summary: Based on the survey of the aforementioned virtual routers, we summarize into the following findings.

- Set-associative table and Cuckoo hash are widely-used hash table designs. They achieve good balance between table utilization, hardware efficiency, and implementation simplicity.

- For various designs, having buckets with 4 to 8 entries (power of 2 entry count makes vector instructions easier) is common. Cache-alignment of a bucket is preferred since it allows for one bucket to be loaded from cache in one shot. For example, OVS uses a bucket entry with 5 keys (not a power of 2) just to fit a bucket within a cache line.

- Hash tables can also be used as caches in software switches (e.g. OVS). When used as a cache a replacement policy is implemented (typically LRU or random) to evict entries.

- Overflow table or extendable buckets hashing in DPDK and OpenContrail provides capacity guarantee by using linked list of extra buckets. Other virtual switches without using overflow table does not provide such guarantee. 


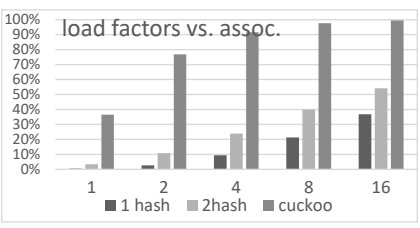

(a)

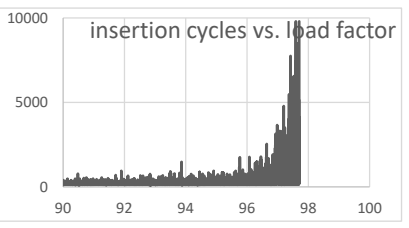

(b)
Figure 1: Table utilization across different structures (a), and insertion cost of Cuckoo hash approaching maximum utilization (b).

- Software optimization techniques are heavily used to hide memory access latency to improve performance (e.g. packet batching, software pipelining, prefetching, manual loop unrolling, etc.)

- Most hash tables support at least one writer thread with multiple reader threads working simultaneously. Cuckoo hashing requires extra care to handle concurrency because of the recursive key displacement process. With one writer, optimistic locking can be used [2]; while with multiple writers, a fine grained locking mechanism should be used on writer threads to improve update performance [13]. Intel® TSX can help in both cases. In [13], it mentions that a fine-grained Intel®TSX based implementation improves writer performance by $20 \mathrm{x}$ comparing to original Cuckoo hashing using spin locks. We will not focus on concurrency implementations in this paper since they are well studied for Cuckoo hash in previous literatures.

\section{DESIGN ANALYSIS}

In this section, we analyze different design options based on our previous summary of hash table designs in various virtual switches. The analysis is based on set-associative table or Cuckoo hashing which are the most widely used hash table designs. We focus on two important aspects of design factors: data structure and software optimizations.

\subsection{Table Organization and Data Structure}

3.1.1 Number of keys per bucket. As previously mentioned, in set-associative hash table each bucket stores multiple keys. In Figure 1(a) we plot the maximum table utilization (i.e. load factor) of each hash table structure with various entry count per bucket.

As shown in figure, in general more entries per bucket leads to higher table utilization. There is clearly diminishing returns after entry count becomes as large as 8 or more especially with Cuckoo hash or 2-hash table.

For Cuckoo hash, it achieves very high utilization even with only 4 entries per bucket. This shows how effective Cuckoo algorithm is. However, Cuckoo hashing could make key insertion extremely costly when approaching maximum table utilization. Figure 1(b) shows how insertion cycle changes vs. utilization. The regular insertion cost increases from around 80 cycles to almost 10000 cycles at worst. This happens after the load factor is as large as $95 \%$. The insertion cost could directly affects how fast the control plane operates when adding new rules to a table for example, which is critical for telecommunication use cases especially.
Conclusion: When table utilization is important, Cuckoo hash should be used over simple set-associative table even with 2 hash functions. 4 or 8 entries per bucket should be big enough. With Cuckoo hash, table should be resized after $90 \%$ utilization to prevent high cost of key insertion.

3.1.2 Separate key storage and cache alignment. To support variable key sizes, many hash tables (e.g. OVS's cmap, DPDK's Cuckoo hash, etc.) store keys in a separate memory location from the main hash table entries, while the hash table itself only stores the signatures of the keys. In this way it is easier to have cache aligned buckets which makes cache access more efficiently. Other designs (e.g. OpenContrail) have opted to store both the signature as well as the full key in hash table entries. The advantage of storing the key inside the hash table itself is that when there is a signature match, there is better hardware cache locality to retrieve the key, since it just sits close by (and probably already prefetched with the underlying hardware prefetcher). However, on the other hand, with the same logic, the hardware prefetcher in the case of the mismatch will bring in useless data in the CPU cache contending for the space that could have been used for other useful data. Clearly, the net effect depends on the traffic pattern and the probability of lookup hits versus miss in the hash table.

To evaluate the effect, we run DPDK packet framework with the two hash table designs (separate vs. adjacent keys) to collect throughput data for all-hit and all-miss lookup cases. Note that both designs are provided by DPDK and they are highly optimized with software pipelining and fully cache aligned for each bucket. This helps to avoid other factors that could affect performance. We tested with 16 and 32-byte key size using 64 byte packets. The DPDK packet framework uses three cores each does RX, TX, and table lookup. Unfortunately, all test shows only 1-3\% throughput difference between two designs. With such small difference, we can't conclude a clear winner with no profound changes in lookup performance for the separate versus adjacent keys. Clearly, an efficient implementation (as discussed in Section 3.2) can hide majority of the memory access latency, which is the major difference between the two schemes.

For cache alignment test, we run DPDK's Cuckoo hash with either cache aligned or not (with or without the cache aligned attribute to the bucket struct). Across various configurations, the batching lookup function improves speed by $6.5 \%-16.7 \%$ with cache aligned buckets.

Conclusion: With efficient software implementations, storing keys separately will not exert much of performance impact since the overhead of extra memory access can be hidden. Meanwhile, cache alignment of each bucket is a necessary optimization.

3.1.3 Hash table based cache. We mentioned that OVS and DPDK both provide libraries to use hash table as a cache. One major difference of using hash table as a cache is that the miss ratio instead of table utilization now becomes the most relevant metric. According to DPDK and OVS, a two-hash set-associative table is commonly used for this purpose. Figure 2(a) shows that with different entries per bucket, how miss ratio changes with 1 million random keys accessing the cache with 1 million entries. To emulate real use cases, the traffic follows power law distribution to simulate locality. The results show that even without Cuckoo 


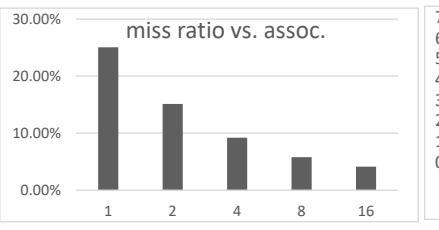

(a)

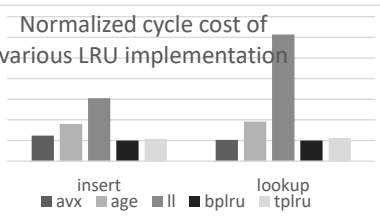

(b)
Figure 2: Miss ratio across various entry count per bucket (a), and LRU implementation comparison (b).

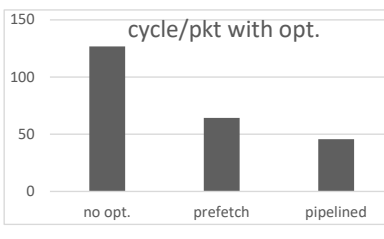

(a)

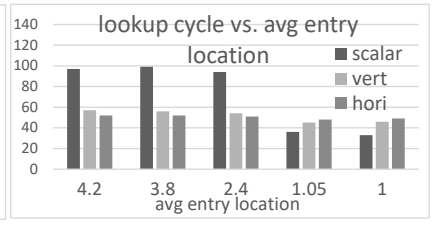

(b)
Figure 3: Software pipelining and prefetching optimizations (a), and comparison of SIMD with scalar implementations (b).

algorithm, the miss ratio could be as low as $4 \%$ with 16 way per bucket.

Conclusion: Hash-table based cache does not need relatively complicated Cuckoo displacement algorithm to achieve low miss ratio. having 8 or 16 ways per bucket is efficient in bringing down miss ratio.

Another aspect is the cache replacement policy. When a bucket is full, a new key has to pick an entry to evict from the bucket. Similar to hardware cache, a LRU policy is commonly preferred. The question is how to implement an efficient LRU policy in software. We compared different LRU implementations with a micro benchmark. They are 1) LL, a linked-list based LRU that most recently accessed entry is put on the head of the list; 2) age based: an age-based LRU that each entry has an age counter and entries that are not recently accessed will have older ages; 3) Bit-PLRU and Tree-PLRU: two pseudo LRU algorithms whose details could be found in [19]; and 4) AVX-LRU, a novel LRU implementation using Intel ${ }^{\circledR}$ AVX2 instruction specifically the permute instruction "_mm256_permute". In one instruction, it can permute the most recently used entry to the head of the bucket. We show the cycle cost comparison in Figure 2(b). The results show that our proposed LRU implementation performs almost the same to the pseudo LRU algorithm, while linked-list is the slowest.

Conclusion: Our proposed Intel $\circledast A V X$ based LRU implementation realizes true $L R U$ with low cost. The pseudo $L R U$ algorithms perform similarly. Other true LRU implementations are too costly and should be avoided in high speed networking applications.

\subsection{Software Optimization Techniques}

3.2.1 Software pipelining and prefetching. From our survey, most of virtual switches take advantages of batched processing for table lookup. Batching means each lookup function call processes a batch of packets. It is intuitive that batching could mitigate the function call overhead across multiple packets, thus increases the total throughput. However, the major benefit of batched lookup is to more easily use prefetch and software pipelining to hide memory access latency. From our test with DPDK, batching-only without any prefetch or pipelining gives little performance benefit. In real life applications, VPP uses software pipelining in most of the processing nodes. There are also researches (e.g. [9]) showing how to automate such optimization in hash table lookups.

In this section, we evaluate a few software techniques including prefetching, pipelining, and loop unrolling based on batching. We tested three implementations with a hash table based software cache in OVS [18] with a batch size of 32 packets. The original implementation does not use any prefetching or software pipelining, the second uses prefetching to prefetch hash table buckets at the beginning of the lookup function for all the 32 packets. The third implementation uses software pipelining to pipeline the lookup process. The results are shown in Figure 3(a). The results indicate that prefetching-only optimization reduces the lookup cost by almost $2 \mathrm{x}$. Software pipelining further reduces the cost by another $40 \%$ over prefetching-only scheme since memory access latency is further hidden.

The third technique we investigate is manual loop unrolling. We did not observe significant performance improvement using manual loop unrolling in our OVS setup. This may be due to 1) big loops does not benefit much from unrolling, and 2) small loops is already unrolled by compilers.

Conclusion: Batching with prefetching and software pipelining is useful to further improve performance. Batching plus prefetching are the most critical optimizations among others. It is worth mentioning that software pipelining reduces the readability of the code which may be not always preferable for open source projects.

3.2.2 Vectorization. Vectorization could be used for key comparison and signature comparison. For key comparison, the mem$c m p()$ in c standard library should already take advantage of vector instructions by modern compilers when key size is large. Programmers usually rely on the compiler to perform the optimization. The signature comparison is the part that could benefit from different implementations. Here we focus on two vectorization implementations: vertical and horizontal. Horizontal vectorization tries to compare one key's signature to all the signatures in one bucket in one shot. Vertical vectorization [17] gathers multiple keys, and compare all keys' signatures with the first (or following) entries of all corresponding buckets. In both cases, each Intel®AVX2 instruction performs eight comparisons (assuming 32-bit signatures), however, the order of the comparison is different.

To compare the performance, we implemented both mechanisms and compare with scalar version and show the result in Figure 3(b). The $\mathrm{x}$-axis is the average location that a matching key is found in a bucket, which indicates how full the hash table is. It is interesting that with various table load, the performance could vary significantly. This results show that different strategies of signature comparisons are optimal for different use cases. When the table is relatively full, a SIMD version of key lookup outperforms significantly, while when the table is relatively empty, it could be beneficial to just use the scalar version. It could be useful when we know the table load. For example, a lot of traffic patterns exhibit 
timing behavior. During late night, the flow table may be relatively less loaded than day time. In such case, the algorithm could adapt accordingly.

Conclusion: There are different vectorization strategies and they are optimal for different use cases. When a table is sparsely filled, a scalar version may perform even better. If affordable, a dynamic logic to choose between vectorization or scalar strategy could be beneficial.

\section{OPTIMIZATION DIRECTIONS}

In this section, we identify two optimization directions for using hash tables in virtual routers. We briefly describe the designs and leave the detailed implementation and evaluation to future work.

Extendable bucket Cuckoo Hash: Many virtual switches, such as in Telecom usages, desire both scalable performance and guaranteed table capacity. The latter is defined as guaranteed number of key insertions regardless of keys' random pattern, even if many keys happen to collide on a few buckets. To satisfy both requirements, we propose a hash table design that combines extendable buckets (i.e. overflow buckets) with Cuckoo hash table. In fact both extendable bucket hash and Cuckoo hash can be found in various virtual switches. The former provides guaranteed table capacity especially in certain corner cases when collisions are severe, while the latter provides high table utilization in normal cases. To get the best of both worlds, our proposal is to employ Cuckoo hash table as the primary hash table while using a linked list of buckets as an overflow table. To implement, We add one pointer in each bucket in primary table to point to extra buckets in overflow table.

This design is to maintain a high utilization primary table with the guaranteed capacity provided by the extendable bucket. With this design, once the load factor becomes high, we expect to observe a faster lookup speed than the traditional extendable table with only one hash function, while achieve deterministic and more efficient insertion than traditional Cuckoo hash table.

Adaptive virtual router: From the survey and discussions, one more conclusion we reached is that there is NO single algorithm or configuration that fits all use cases, especially when the traffic patterns and rules in a virtual router commonly change over time.

One optimization is to design an adaptive virtual router that adaptively chooses an optimal option during run time from various configurations and algorithms it provides. At the beginning, the default option is used (same as current design). The router continuously monitors the performance (e.g., throughput, packet drop rate, etc.). If the performance decreases below a certain threshold, the router will enters a "trial and rank" phase. During this phase, the adaptive algorithm tries various potential hash tables with different algorithms or configurations. The new performance is recorded and ranked, eventually the highest ranking option is used for the next period of time. This mechanism not only applies to hash tables but also applies to the general classification algorithm of the virtual router. The trial and ranking process could be replaced by other more time efficient approaches. such as a "table dueling" approach when multiple cores perform software switching. With the table dueling approach, each packet processing core uses one distinct data structure and the performance of all the cores are compared against each other. The winner core's data path algorithm will be duplicated to all the other cores during the next epoch.

\section{CONCLUSIONS AND FUTURE WORK}

Hash table is one of the most performance critical data structure in kernel-bypass virtual switches. In this paper, we investigated various hash table implementations and usages. We analyzed the performance impact of various software implementations and algorithms. We envision the analysis could provide guidelines to software router designers on designing optimal hash tables.

Based on the understanding, we also proposed novel designs such as Intel® AVX based LRU cache and identified two optimization directions, which will be our future work.

\section{ACKNOWLEDGEMENT}

We thank our colleagues Xiangyang Guo, Pablo De Lara Guarch, Bruce Richardson, and Venky Venkatesan for their insights and comments which help the work. We also thank all the anonymous reviewers who provide valuable reviews for us to improve the paper.

\section{REFERENCES}

[1] dpdk.org. online. DPDK main page. dpdk.org. (online). [accessed 16-March-2018].

[2] Bin Fan, David G. Andersen, and Michael Kaminsky. 2013. MemC3: Compact and Concurrent MemCache with Dumber Caching and Smarter Hashing. In Proceedings of the 10th USENIX Conference on Networked Systems Design and Implementation (nsdi'13). 371-384.

[3] Bin Fan, Dave G. Andersen, Michael Kaminsky, and Michael D. Mitzenmacher. 2014. Cuckoo Filter: Practically Better Than Bloom. In Proceedings of the 10th ACM Int. on Conf. on Emerging Networking Experiments and Technologies (CoNEXT '14). ACM, 75-88.

[4] fd.io. online. VPP main page. https://wiki.fd.io/view/VPP. (online). [Online; accessed 16-March-2018].

[5] Dimitris Fotakis, Rasmus Pagh, Peter Sanders, and Paul Spirakis. 2005. Space Efficient Hash Tables with Worst Case Constant Access Time. Theory of Computing Systems 38, 2 (01 Feb 2005), 229-248.

[6] Sangjin Han, Keon Jang, Aurojit Panda, Shoumik Palkar, Dongsu Han, and Sylvia Ratnasamy. 2015. SoftNIC: A Software NIC to Augment Hardware. Technical Report. EECS Department, University of California, Berkeley.

[7] Maurice Herlihy, Nir Shavit, and Moran Tzafrir. 2008. Hopscotch Hashing. In Proceedings of the 22Nd International Symposium on Distributed Computing (DISC '08). 350-364.

[8] https://tungstenfabric.io/. online. Tungsten Fabric main page. https:// tungstenfabric.io/. (online). [accessed 6-June-2018].

[9] Anuj Kalia, Dong Zhou, Michael Kaminsky, and David G. Andersen. 2015. Raising the Bar for Using GPUs in Software Packet Processing. In 12th USENIX Symposium on Networked Systems Design and Implementation (NSDI 15).

[10] Donald E. Knuth. 1997. The Art of Computer Programming, Volume 1 (3rd Ed.): Fundamental Algorithms.

[11] Per-Ake Larson. 1988. Dynamic Hash Tables. Commun. ACM 31, 4 (April 1988), 446-457.

[12] N. Le Scouarnec. 2017. Cuckoo++ Hash Tables: High-Performance Hash Tables for Networking Applications. ArXiv e-prints (Dec. 2017). arXiv:cs.NI/1712.09624

[13] Xiaozhou Li, David G. Andersen, Michael Kaminsky, and Michael J. Freedman. 2014. Algorithmic Improvements for Fast Concurrent Cuckoo Hashing. In Proceedings of the Ninth European Conference on Computer Systems (EuroSys '14). Article 27, 14 pages.

[14] Rasmus Pagh and Flemming Friche Rodler. 2001. Cuckoo hashing. In fournal of Algorithms.

[15] Rina Panigrahy. 2005. Efficient Hashing with Lookups in Two Memory Accesses. In Proceedings of the Sixteenth Annual ACM-SIAM Symposium on Discrete Algorithms (SODA '05). 830-839.

[16] Ben Pfaff, Justin Pettit, Teemu Koponen, Ethan Jackson, Andy Zhou, Jarno Rajahalme, Jesse Gross, Alex Wang, Joe Stringer, Pravin Shelar, Keith Amidon, and Martin Casado. 2015. The Design and Implementation of Open vSwitch. In 12th USENIX Symposium on Networked Systems Design and Implementation (NSDI 15). 117-130.

[17] Orestis Polychroniou, Arun Raghavan, and Kenneth A. Ross. 2015. Rethinking SIMD Vectorization for In-Memory Databases. In Proceedings of the 2015 ACM SIGMOD International Conference on Management of Data (SIGMOD '15). 14931508.

[18] Yipeng Wang, Tsung-Yuan Charlie Tai, Ren Wang, Sameh Gobriel, Janet Tseng, and James Tsai. 2017. Optimizing Open vSwitch to Support Millions of Flows. In 2017 IEEE Global Communications Conference. 1-7. 
[19] WIKIPEDIA. online. Wiki page on pseudo LRU. https://en.wikipedia.org/wiki/ Pseudo-LRU. (online). [accessed 16-March-2018].

[20] www.opencontrail.org. online. OpenContrail main page. www.opencontrail.org. (online). [accessed 16-March-2018].

[21] Dong Zhou, Bin Fan, Hyeontaek Lim, Michael Kaminsky, and David G. Andersen. 2013. Scalable, High Performance Ethernet Forwarding with CuckooSwitch. In Proceedings of the Ninth ACM Conference on Emerging Networking Experiments and Technologies (CoNEXT '13). 97-108. 